\title{
Neonatal Society
}

\section{Meeting held on 2 February 1978 at St Thomas's Hospital, London}

\section{Communications}

Group B haemolytic streptococcal carrier rate in pregnant mothers and their newborn infants. J. E. Cree and B. T. Thom (introduced by T. P. Mann). Neonatal Unit and Microbiology Department, Royal Sussex County Hospital, Brighton.

Umbilical cord water content. J. D. Baum and K. Sloper. Department of Paediatrics, John Radcliffe Hospital, Oxford.

Role of catecholamines in lung liquid absorption at birth. R. E. Olver and D. V. Walters. Department of Paediatrics, University College Hospital Medical School, London.

Mechanism of increased lung wash content of phosphatidylcholine produced by oxotremorine in neonatal rabbits. M. M. Abdellatif and M. Hollingsworth (introduced by J. A. Davis). Departments of Child Health and Pharmacology, Materia Medica and Therapeutics, University of Manchester.

Continuous measurement of oxygen saturation of haemoglobin in sick newborn infants. G. A. Gregory, R. H. Phibbs, and A. R. Wilkinson (introduced by J. P. M. Tizard). Departments of Pediatrics and Anaesthesia, and Cardiovascular Research Institute, University of California, San Francisco, USA.

Inappropriate secretion of antidiuretic hormone in preterm infants treated by continuous positive airway pressure and artificial ventilation. P. Czernichow, G. Moriette, R. Pomarede, and J. P. Relier (introduced by A. Minkowski). Hôpital Port-Royal and Hôpital des Enfants Malades, Paris, France.
Apparent cerebral blood flow in neonatal respiratory disease. R. W. I. Cooke, P. Howat, and P. Rolfe. Department of Paediatrics, John Radcliffe Hospital, Oxford.

Cerebral blood flow and metabolism in the newborn calf. R. M. Gardiner (introduced by N. R. C. Roberton). Physiological Laboratory, University of Cambridge.

Hats and heat loss in the newborn infant. J. K. Stothers and R. M. Warner (introduced by K. W. Cross). Neonatal Research Group, Department of Physiology, The London Hospital Medical College, London.

Simplified accurate technique for the measurement of oxygen consumption. J. M. Evans, O. P. Gray, B. M. Holland, and C. A. J. Wardrop. Departments of Anaesthetics, Child Health, and Haematology, University Hospital of Wales, Cardiff.

1000 consecutive neonatal electrocardiograms. D. A. Chamberlain, R. W. A. Jones, B. R. Lambert, L. M. Rabb, and C. Sharp (introduced by T. P. Mann). Royal Sussex County Hospital, Brighton.

Further studies into the nature of cardiac arrhythmias in the newborn infant. P. Johnston, E. A. Shinebourne, and D. P. Southall (introduced by I. C. S. Normand). Departments of Paediatrics, Dorset County Hospital, Dorchester, and Brompton Hospital, London.

Fluid intake and weight recovery in breast- and bottlefed infants. P. E. Culley, P. Milan, and B. Wood. Birmingham Maternity Hospital, Birmingham. 